\title{
Optical Microstructure Design Optimization for Display Backlighting
}

\author{
Chi-Chang Hsieh ${ }^{1}$, Yan-Huei $\mathrm{Li}^{2}$ \\ ${ }^{1}$ Department of Mechanical and Automation Engineering, National Kaohsiung \\ First University of Science and Technology, Taiwan \\ ${ }^{2}$ Instiute of Engineering Science and Technology, National Kaohsiung \\ First University of Science and Technology, Taiwan \\ Email: cchsieh@nkfust.edu.tw, yanhuei.li@gmail.com
}

Received September 6, 2013; revised October 22, 2013; accepted November 8, 2013

Copyright (C) 2013 Chi-Chang Hsieh, Yan-Huei Li. This is an open access article distributed under the Creative Commons Attribution License, which permits unrestricted use, distribution, and reproduction in any medium, provided the original work is properly cited.

\begin{abstract}
This study proposes an innovative design method for functional optical film microstructures used in displays and applies this design to LCD backlighting to replace multi-layer optical film functional integration with composite optical film. We design a novel optical film microstructure based on light uniformity and wide-angle lumination distribution and determine the optimal optical microstructure parameters by combining the global optimization of a genetic algorithm with ray tracing. The purpose of this study is to develop substitutes for traditional multi-layer prism brightness enhancers and light-diffusing film stacks and to examine the structural changes during calculations and summarize the characteristics. In this study, we focus on determining the optimal light uniformity of new optical film microstructures. The seven-inch LED backlight module of the method proposed in this study achieved $94.59 \%$ uniformity and $168^{\circ}$ lumination distribution while reducing thickness by $66 \%$ to $82 \%$ compared to a traditional multi-layer optical film stack.
\end{abstract}

Keywords: Optical Film; Microstructure; Display; Backlight

\section{Introduction}

In recent years, green energy-saving and ultra-thin displays have become the demand for the mainstream market. Therefore, backlight modules with extremely high cost and assembling ratios have become fiercely contested items among major manufacturers because of the technological innovation requirements. Multiple optical films with various effects are stacked in layers in backlight modules to focus the light energy and equalize the light distribution. However, this increases the cumulative thickness and overall cost of backlight modules. Currently, backlight modules for displays typically comprise brightness enhancing films with prismatic structures to enhance luminance in the normal direction. However, this approach is likely to cause significant declines in luminance at minor angles away from the normal direction. Therefore, diffusion films are commonly added to reduce the significant decline in luminance and improve uniformity. However, this reduces the axial luminance. During this back-and-forth tuning process, the backlight module tends to become excessively thick because of the addi- tion of extra optical films, which also dissipate the light energy. Thus, the goal of this study was to design a compound optical film with an innovative structure emphasizing light uniformity. The aim was to widen the luminance distribution viewing angle and resolve the uneven luminance in the central viewing angles of older structures to achieve one-to-two or one-to-many performances, replace the traditional process of stacking multiple optical films together, reduce the thickness of backlight modules, design an easy-to-manufacture structure, and effectively reduce costs.

This study employed a genetic algorithm (GA) as the model computational framework. GAs were first proposed in 1975 by Holland. Using the GA method enables a variety of problems to be converted into genotypes [1]. In 2009, Li et al. combined a neural network and realvalued GA to optimize the design of the bottom structure of a light guide plate. In their study, the luminance uniformity reached $92.09 \%$ and showed greater accuracy than that of results provided by the modeling software ASAP [2]. In 2010, Yamada et al. employed an evolutionary algorithm (EA) to automatically generate a set of 
Fresnel lenses of varying geometries and densities. This method achieved the highly uniform flux concentration required for the design of photovoltaic cells [3].

Genetic algorithm has less been applied in backlight module with a light guide structure design. In 2008, Fan et al. applied genetic algorithms to design LED lens in direct type LED Backlight Unit to achieve high uniformity [4]; In 2009, Li et al. applied genetic algorithm to optimize the distribution density of the prism pattern to improve the uniformity of the light guide plate [2]. In 2013, Kim et al. applied genetic algorithm to numerical model of a planar prism LED luminaire, and to the analysis of the best angle prism [5]. This study proposes an innovative design and optimize structure applied Genetic algorithm, which can achieve high uniformity of the illuminance distribution with a large angle.

\section{Design Analysis of optical Film Structure}

\subsection{Configuration of Backlight Modules}

This study used sidelight-type backlight modules with 7-in no-wedge angle light guide plates with the following dimensions: $92 \mathrm{~mm}$ in width, $158 \mathrm{~mm}$ in length, and 2.5 $\mathrm{mm}$ in thickness. The material used was PMMA with a refractory index of 1.49 . The reflectivity was approximately $95 \%$. The distance between the light exit face of the light guide plate and the receiver board was $0.45 \mathrm{~mm}$. Only the no-wedge angle light guide plates contained concave hemispheric structures, the rest were all unstructured optical surfaces. The diameter of the structure was $0.1 \mathrm{~mm}$. The distance between the structures was $1 \mathrm{~mm}$ in both the $\mathrm{X}$ and $\mathrm{Y}$ directions. The light source was an LED, specifically item number NESW020A from NICHA, which emits $3.7 \mathrm{~lm}$ with a $20 \mathrm{~mA}$ electrical supply at a light-emitting angle of $120^{\circ}$. The light pattern was close to a Lambertian distribution. Additionally, 7 LEDs were arranged equidistantly along the short edge of the light guide plate.

\subsection{Design Method for the optical Structure Geometry}

The structural geometry concept of this study was based on achievements in surface microstructure design for LED packaging completed by our laboratory [6,7]. Results from the research have indicated that stepped structures have light focusing effects. Applying this characteristic to the design of optical films has a potential to provide excellent performances. The original stepped structure (shortened to original structure hereafter) in the patent awarded to our laboratory in 2008 for composite optical film can achieve a wider luminance distribution throughout the viewing angle [8]. However, the axial luminance remains inferior to that of a prismatic sheet combined with a diffusion film. Because our laboratory had already generated a conceptual structure, which had room for improvement, we focused on introducing lightfocusing novel structures to enhance the brightness and performance. Therefore, this study used the concept of geometric shape combinations to combine the prismatic structure providing brightness-enhancing effects with stepped structures providing broader luminance distributions throughout the viewing cone, in the hopes that multiple performance improvements could be achieved. Figure 1 shows the design concept diagram for combining geometric shapes. Square structures were introduced into the original prismatic structure, forming stepped geometric structures similar to oblique prisms. Novel structures were created through equidistant division, changing angles, or changing the structural width and height. This enabled the structural parameters to be defined, which facilitated the experimental process to identify optimal solutions.

The shape and dimension of the optical film structure refer to the structural dimensions of the prismatic brightness-enhancing film 3M BEFII 90/50. The structural shape of 3M BEF 90/50 comprises equilateral triangles $23 \mu \mathrm{m}$ in height, with a tip angle of $90^{\circ}$, and arranged in parallel rows. The novel geometrical structure in this study was based on this geometry and further divided equidistantly into 16 sections in the horizontal direction and 8 sections in the vertical direction. The equidistant division lines along the edges of the prismatic surfaces were joined to form the stepped structure proposed in this study. Figure 2 shows a conceptual diagram of the design of this novel structure.

\subsection{Defining the structural Parameters of the optical Geometry}

Figure 3 shows a diagram that defines the structural shape of the stepped structure. In this figure, W represents the base width of a single structure. The width of each exposed step was $\mathrm{W} / 16$. $\mathrm{H}$ represents the overall height of the structure, and the height for each step was $\mathrm{H} / 8$. $\theta$ represents the angle from the edge of the stepped structure to the horizontal plane. When $\theta$ was $45^{\circ}$, the

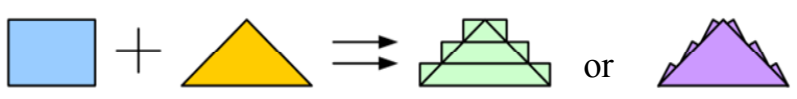

Figure 1. Design concept for the combination of geometries.

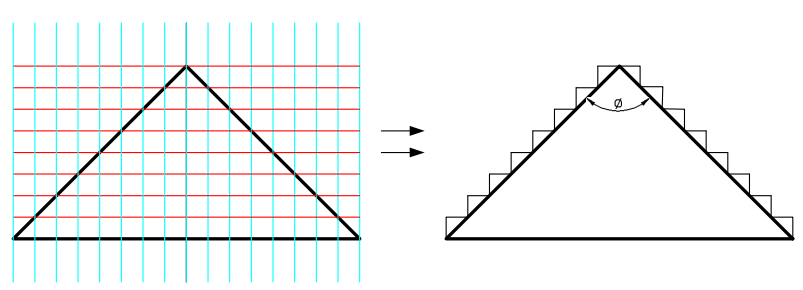

Figure 2. Design concept for the new type of structure. 


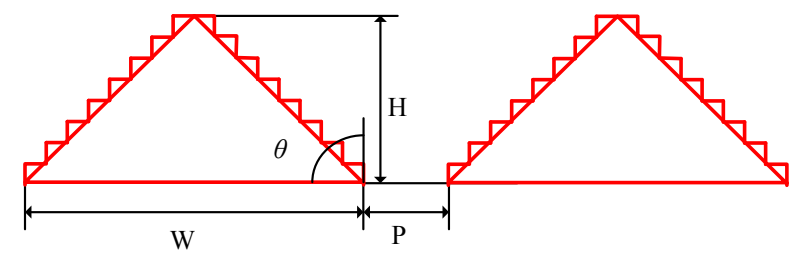

Figure 3. The external dimensions of the stepped structure.

formed structure was a triangular prism. $\mathrm{P}$ represents the distance between each structure. The 8-stepped structural arrays could be defined completely using these 4 parameters. Once the parameters were defined, the machining limit or the size of commercially available optical film sheets was used as the boundary conditions for the reasonable ranges of structural parameters. This mitigates the manufacturing difficulties caused by excessively small structures, or an inability to effectively downscale the thickness of backlight modules because of excessively large structures.

\subsection{Methods for Measuring Uniformity}

This study used the 9-point VESA measurement method as a reference for uniformity measurements of the 7-inch backlight module. The luminance value of each point was first measured, then the 9-point VESA measurement method commonly employed throughout the industry was applied [9]. The minimum and maximum luminance values of backlight modules measured using the 9-points were shown as percentages, which were the level of uniformity of the panel. The uniformity formula is shown below:

$$
\text { Uniformity }=100 \% \times \frac{\mathrm{L}_{\min }}{\mathrm{L}_{\max }}
$$

\subsection{Full Width at Half Maximum}

In 2009, Sang-HoonBaik used the full width at half maximum (FWHM) to define the angular luminance distribution of optical films to determine the size of the view angle [10], which was then examined against an orthogonally arranged dual prismatic sheet for performance comparison. This study referenced this definition of view angle to define the view angle of the angular luminance distribution curve. This luminance angle differs from the commercially available viewable angle determined by the level of contrast. For TFT-LCDs, a higher luminance angle is less likely to cause uneven brightness and luminance declines when viewed from multiple angles.

\section{Results and Discussion}

\subsection{Results from the genetic Algorithm}

Figure 4 is a fitness function curve for the GA. The fig- ure shows that by generation 33, the difference between chromosomes was sufficiently minor that neither mating nor mutation could achieve superior solutions. The iterating computation using the optimal value obtained in this GA was stopped at generation 100 because the termination criteria were satisfied. Figure 5 shows the optimized exterior structure through a global search method using the GA.

\subsection{Analysis of structural form and Performance}

\subsubsection{Discussion on the Relationship between structural form and View Angle Luminance Distribution}

Table 1 shows the interval combinations and uniformity values for intervals of $0.03 \mathrm{~mm}$, with uniformity values above $92 \%$. Because of the many combinations, only combinations with uniformity values above $92 \%$ were included in the examination. Based on the 26 combinations of structural shapes and light trace charts, we found that the structural shapes could be roughly classified into 3 types. The various shape types are shown in Figure 6. TYPE 1 comprises prismatic structures, TYPE 2 comprises step-shaped structures with larger side angles, and TYPE 3 comprises step-shaped structures with smaller side angles.

Figure 6 shows that the prismatic structure of TYPE 1 shapes characteristically corrects light rays at greater angles of deviation from the normal line to within plus or minus $30^{\circ}$ from the center. Luminance at the central view angles is higher and more concentrated than the lumi-

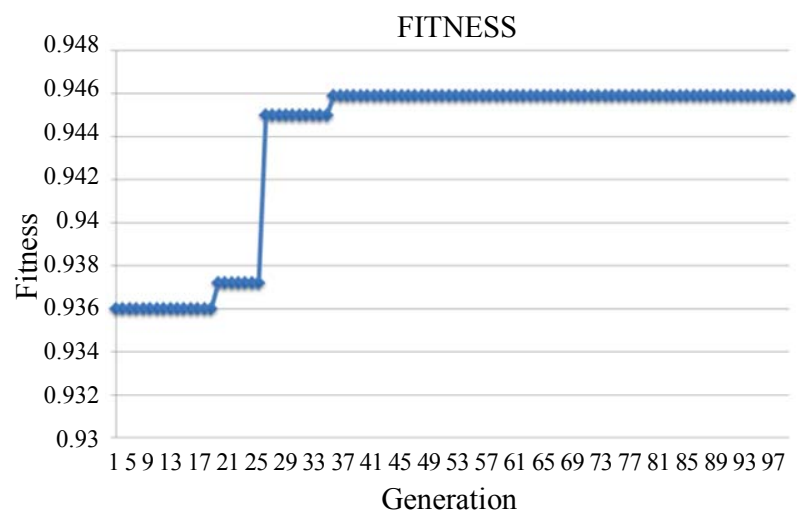

Figure 4. Fitness function curve of the genetic algorithm.

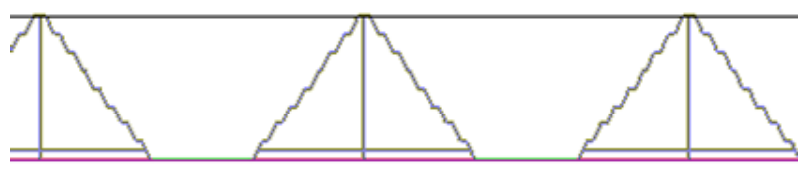

Figure 5. The optimal structural shape obtained using the GA. 
Table 1. Parameter combinations with intervals of $0.03 \mathrm{~mm}$ and respective uniformity values.

\begin{tabular}{|c|c|c|c|c|c|}
\hline Width & Height & Angle & Interval & Uniformity (\%) & Generation \\
\hline 0.858 & 0.265 & 79.131 & 0.030 & 92.01 & G34 \\
\hline 0.061 & 0.038 & 80.520 & 0.030 & 92.02 & G50 \\
\hline 0.061 & 0.038 & 85.367 & 0.030 & 92.03 & G59 \\
\hline 0.061 & 0.038 & 85.168 & 0.030 & 92.04 & G74 \\
\hline 0.061 & 0.038 & 66.625 & 0.030 & 92.07 & G69 \\
\hline 0.061 & 0.019 & 65.000 & 0.030 & 92.09 & G42 \\
\hline 0.122 & 0.038 & 65.000 & 0.030 & 92.09 & G43 \\
\hline 0.061 & 0.038 & 72.855 & 0.030 & 92.09 & G69 \\
\hline 0.061 & 0.031 & 65.000 & 0.030 & 92.12 & G65 \\
\hline 0.061 & 0.038 & 87.724 & 0.030 & 92.12 & G71 \\
\hline 0.137 & 0.038 & 65.000 & 0.030 & 92.13 & G62 \\
\hline 0.061 & 0.021 & 65.000 & 0.030 & 92.35 & G55 \\
\hline 0.061 & 0.038 & 74.936 & 0.030 & 92.35 & G62 \\
\hline 0.106 & 0.038 & 65.000 & 0.030 & 92.35 & G62 \\
\hline 0.061 & 0.038 & 84.619 & 0.030 & 92.38 & G63 \\
\hline 0.636 & 0.300 & 65.000 & 0.030 & 92.76 & G39 \\
\hline 0.061 & 0.021 & 65.000 & 0.030 & 92.81 & $\mathrm{G} 72$ \\
\hline 0.083 & 0.038 & 65.000 & 0.030 & 92.84 & G58 \\
\hline 0.061 & 0.026 & 65.000 & 0.030 & 92.85 & G41 \\
\hline 0.064 & 0.038 & 65.000 & 0.030 & 92.86 & G99 \\
\hline 0.161 & 0.096 & 65.000 & 0.030 & 92.92 & G45 \\
\hline 0.090 & 0.038 & 65.000 & 0.030 & 93.27 & G53 \\
\hline 0.061 & 0.064 & 65.000 & 0.030 & 93.51 & G51 \\
\hline 0.136 & 0.038 & 65.000 & 0.030 & 93.63 & G67 \\
\hline 0.061 & 0.035 & 65.000 & 0.030 & 93.88 & G42 \\
\hline 0.061 & 0.040 & 65.000 & 0.030 & 94.59 & G33 \\
\hline
\end{tabular}

nance at larger view angles, ranging between 180 nit and 190 nit. The luminance view angle was measured as $80^{\circ}$ using the FWHM measurement method.

TYPE 2 comprised stepped structures with larger side angles. The light trace chart of TYPE 2 shapes in Figure 6 shows that the overall luminance distribution of these structures was generally wider, with weaker luminance in the central view angles, ranging between approximately 150 nit and 160 nit. However, the luminance view angle was wider at approximately $178^{\circ}$. This indicates that the characteristic of TYPE 2 structures is a wider viewing angle from the light source.

TYPE 3 also comprises stepped structures, but these structures have smaller side angles that are generally below $80^{\circ}$. The light trace chart of TYPE 3 structures in Figure 6 shows that, similar to that of TYPE 2 structures, the light travel angle is slightly enlarged, causing the light to proceed in directions with greater viewing angles. When light travels through the side inclination angles of stepped structures, the law of refraction guides the light toward more central viewing angles, achieving the same light-focusing effect as that of prismatic structures. This causes the view angle luminance distribution chart for TYPE 3 structures to show higher central luminance at between approximately 180 nit and 190 nit. The luminance values for larger viewing angles are significantly higher than the values for prismatic structures with viewing angles of approximately $168^{\circ}$. These comparison results indicate that stepped TYPE 3 structures have the light-focusing characteristic of prismatic structures with the wide-angle characteristic of TYPE 2 stepped structures. This improved the low luminance value in central viewing angles. With the structural parameter combinations derived from the GA, an excellent uniformity of $94.59 \%$ was obtained.

\subsubsection{Comprehensive Comparisons}

Figure 7 is a comprehensive comparison chart of various optical film combinations and viewing angle luminance distributions. The results were used to evaluate the luminance distribution of various types of optical films in various angles. Compared to optical films with the original stepped structure, regardless of whether the original stepped structure had a viewing angle approximately $10^{\circ}$ wider than that of the GA structure, the axial luminance of the GA structure was $17.2 \%$ higher than that of the original step structure, with a $1.99 \%$ increase in uniformity.

Backlight modules generally use light-focusing prismatic structures in brightness-enhancing films. However, uneven brightness occurs at greater viewing angles with extremely steep declines. Therefore, another diffusion film is typically used to equalize the light distribution and reduce the significant decline in luminance. Light smoothing also improves the uniformity of backlight modules. As shown in Figure 6, the viewing angle for the GA structure was $67^{\circ}$ wider than that of a single brightness enhanced film (BEF) with increased membrane, and shows a significant difference in luminance gap beyond the plus or minus $50^{\circ}$ viewing angels.

Regarding uniformity, comparing the uniformity results obtained using the GA and the addition of diffusion film to prismatic sheets showed that the $94.59 \%$ of the GA structure was the optimum value. This uniformity value was also $2.52 \%$ higher than that of adding a diffusion film to a single BEF, and $3.48 \%$ higher than that of adding a diffusion film to dual BEFs. 


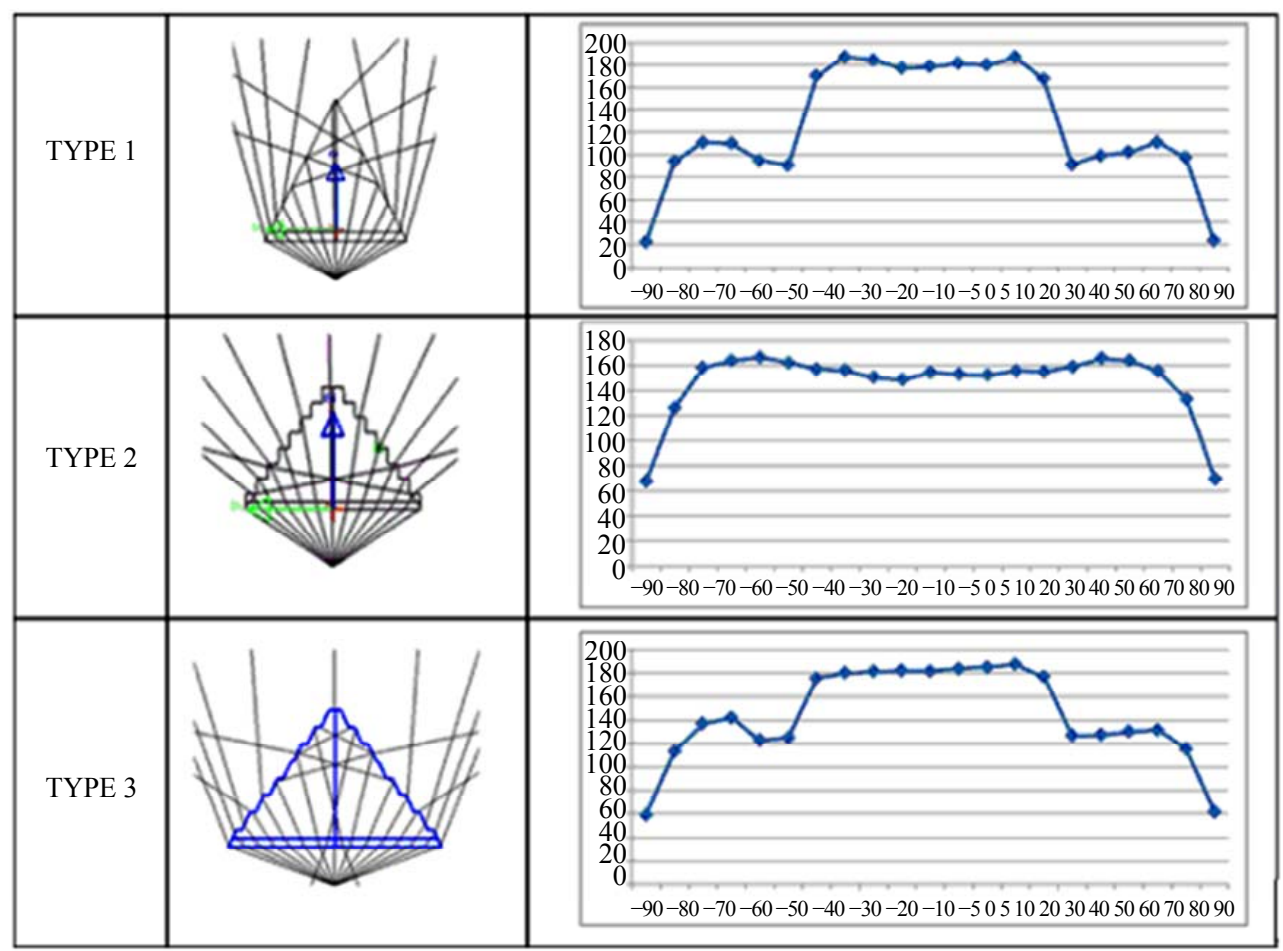

Figure 6. Comparison of the light trace charts and viewing angle luminance distribution curves for each type of structural form.

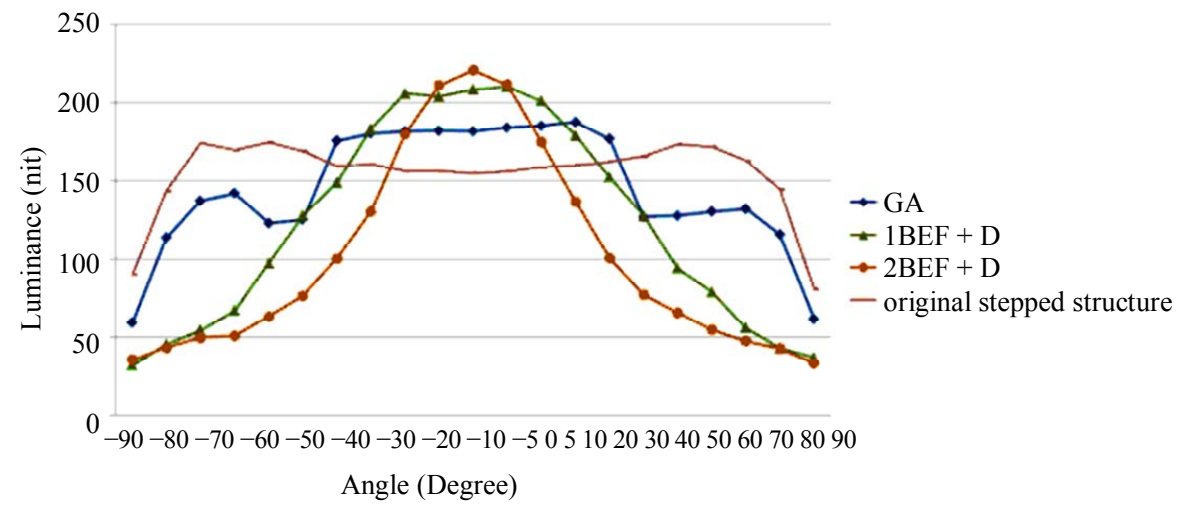

Figure 7. The viewing angle luminance distribution curve for various groups.

These comparisons show that the GA structures provide performances that are comparable or even superior to those of commercially available optical films. Regarding the number of optical films used, the GA structure optical films achieved the optimal performance with a single layer. Compared to the $100 \mu \mathrm{m}$ to $200 \mu \mathrm{m}$ thickness and high cost of diffusion films commonly used in the market, the height of the GA structure was merely 41 $\mu \mathrm{m}$. The prismatic sheet plus diffusion film measured $123 \mu \mathrm{m}$ to $223 \mu \mathrm{m}$ in thickness. The GA structure reduced the thickness by $66 \%$ to $82 \%$. Thus, the film thickness and costs were substantially reduced, and various issues associated with stacking multiple optical films together were also avoided.

\section{Conclusion}

In this study, we proposed the idea of combining geometric shapes to design new compound function optical microstructures. Real-valued coding was used to introduce the GA, which then identified a set of optimized optical microstructures with superior uniformity at $94.59 \%$. Conclusions based on analysis of the modeling results and consolidated data are provided below:

1) The height of the designed structure was $40 \mu \mathrm{m}$, which is only $17 \mu \mathrm{m}$ more than that of the prismatic $\mathrm{M}$ BEFII 90/50 structure. Because the addition of diffusion films with a thickness between $100 \mu \mathrm{m}$ and $200 \mu \mathrm{m}$ is not required to achieve good uniformity, a good lumi- 
nance view angle, and a good axial luminance, the overall thickness is reduced by approximately $66 \%$ to $82 \%$. By only requiring the use of a single optical film, the structural alignment and abrasion damage problems involved in stacking multiple optical films are avoided, and the light source use rate is improved through the reduced dissipation of light energy. Thus, the goal of reducing the thickness of the backlight module through the design of a compound optical film is achieved.

2) The influence of the shapes on the angular luminance distribution can be observed by classifying the structural forms. The results indicate that the prismatic structure is extremely effective for focusing light, but a steep angular luminance decline occurs at greater viewing angles. Additionally, the width of the viewing angle is insufficient. Stepped structures with larger side angles are more effective for dispersing light, showing greater viewing angles. However, the brightness at central viewing angles tends to decline. The GA structure was a stepped structure with smaller side angles. When light passes through the higher part of the stepped structure, the prismatic collimation effect was exhibited. The light was dispersed when passed through the wide part. Therefore, this structure can combine these two features to achieve the optimal design of an optical microstructure.

3) After optimization, the GA structure proposed in this study had a luminance viewing angle of approximately $168^{\circ}$. Although it was approximately $10^{\circ}$ smaller than that of the original structure, the axial luminance was increased by $17.2 \%$, which resolved the problem of low central luminance in the original structure. The uniformity was also approximately $1.99 \%$ higher than that of the original structure.

Comparing the GA structure optimized in this study with the traditional pairing of prismatic sheets and diffusion films, the viewing angle of the GA structure was $67^{\circ}$ better than that of the $1 \mathrm{BEF}+$ diffuser, and $118^{\circ}$ greater than that of the $2 \mathrm{BEF}+$ diffuser configuration. Regarding uniformity, the GA structure was superior to both film combinations, increasing uniformity by $2.52 \%$ and $3.45 \%$, respectively. The results show that using a single sheet of GA-structured optical film effectively improves the uniformity to provide good luminance viewing angles without requiring diffusion films.

\section{REFERENCES}

[1] J. H. Holland, "Adaptive in Natural and Artificial Systems," University of Michigan Press, Ann Arbor, 1975.

[2] C. J. Li, Y. C. Fang and M. C. Cheng, "Study of Optimization of an LCD Light Guide Plate with Neural Network and Genetic Algorithm," Optics Express, Vol. 17, No. 12, 2009, pp. 10177-10188.

http://dx.doi.org/10.1364/OE.17.010177

[3] N. Yamada and T. Nishikawa, "Evolutionary Algorithm for Optimization of Non-Imaging Fresnel Lens Geometry," Optics Express, Vol. 18, No. S2, 2010, pp. A126A132. http://dx.doi.org/10.1364/OE.18.00A126

[4] Y. H. Fan, Y. L. Liao, R. J. Chen, S. K. Chang and J. C. T. Hsieh, "Optimal Design of LED Backlight Unit with Double-Triangular Structure Using a Genetic Algorithm," Fourth International Conference on Natural Computation, Jinan, 25-27 August 2008, pp. 625-629.

[5] Y. S. Kim, A. S. Choi and J. W. Jeong, "Applying Micro Genetic Algorithm to Numerical Model for Luminous Intensity Distribution of Planar Prism LED Luminaire," Optics Communications, Vol. 293, 2013, pp. 22-30. http://dx.doi.org/10.1016/j.optcom.2012.11.017

[6] P. Y. Tsai, C. C. Hsieh and Y. H. Li, "Application of a Novel Focused Microlens to Light-Emitting Diode Packaging," Journal of Micro/Nanolithography, MEMS, and MOEMS, Vol. 9, No. 3, 2010, Article ID: 033012. http://dx.doi.org/10.1117/1.3487740

[7] C. C. Hsieh, P. Y. Tsai, Y. H. Li and C. W. Fan, "Investigation of the Influence of Structural Parameters of a Fresnel-Type Lens on the Narrowing of Light-Emitting Diode Light Beams," Journal of Microlithography, Microfabrication, and Microsystem, Vol. 10, No. 3, 2011, pp. 1-9.

[8] C. C. Hsieh, C. K. Yen and C. Y. Wang, "Composite Optical Film," Patent M330483, 2008.

[9] P. Downen, "A Closer Look at Flat-Panel-Display Measurement Standards and Trends," Information Display, Vol. 22, 2006, pp. 16-21.

[10] S. H. Baik, S. K. Hwang, Y. G. Kim, G. Park, J. H. Kwon, W. T. Moon, S. H. Kim, B. K. Kim and S. H. Kang, "Simulation and Fabrication of the Cone Sheet for LCD Backlight Application," Journal of the Optical Society of Korea, Vol. 13, No. 4, 2009, pp. 478-483. http://dx.doi.org/10.3807/JOSK.2009.13.4.478 